\title{
Optimization aspects of the new nELBE photo-neutron source
}

\author{
A. Ferrari ${ }^{1,2}$, R. Beyer ${ }^{2}$, E. Birgersson ${ }^{2}$, J. Claussner ${ }^{2}$, E. Grosse ${ }^{2,3}$, R. Hannaske ${ }^{2}$, A. Junghans ${ }^{2}$, \\ M. Kempe ${ }^{2}$, T. Kögler² ${ }^{2}$ R. Massarczyk ${ }^{2}$, A. Matič², K.-D. Schilling ${ }^{2}$, G. Schramm² , R. Schwengner ${ }^{2}$, \\ A. Wagner ${ }^{2}$, F.P. Weiss ${ }^{1}$, and D. Yakorev $^{2}$ \\ 1 Institut für Sichereitsforschung, FZD Dresden-Rossendorf, PF 510119, 01314 Dresden, Germany \\ 2 Institut für Strahlenphysik, FZD Dresden-Rossendorf, PF 510119, 01314 Dresden, Germany \\ 3 Institut für Kern- und Teilchenphysik, Technische Universität Dresden, 01062 Dresden, Germany
}

\begin{abstract}
The nELBE beamline at Forschungszentrum Dresden-Rossendorf (FZD) provides intense neutron beams by stopping primary electrons in a liquid lead target, where neutrons are produced by bremsstrahlung photons via $(\gamma, \mathrm{n})$ reactions. With the aim to increase the neutron yield through the enhancement of the electron beam energy (from the current $40 \mathrm{MeV}$ limit up to $50 \mathrm{MeV}$ ), as well as to minimize several sources of background that are presently affecting the measurements, a new neutron beam-line and a new, larger neutron experimental room have been designed. The optimization of the neutron/photon ratio, the minimization of the backscattered radiation from the walls and the possibility to have better experimental conditions are the main advantages of the new design. To optimize the beamline, extensive simulations with the particle interaction and transport code FLUKA have been performed. Starting from the primary electron beam, both the photon and neutron radiation fields have been fully characterized. To have a cross-check of the results, the calculated values of the neutron yields at different energies of the primary beam have been compared both with an independent simulation with the MCNP code and with analytical calculations, obtaining a very satisfactory agreement at the level of few percent. The evaluated radiation fields have been used to optimize the direction of the new neutron beamline, in order to minimize the photon flash contribution. A general overview of the new photo-neutron source, together with all the steps of the optimization study, is here presented and discussed.
\end{abstract}

\section{Introduction}

The superconducting LINAC at the radiation source ELBE (Electron Linear Accelerator with high Brilliance and Low Emittance) at FZD [1] produces high brilliance electron beams with energies up to $40 \mathrm{MeV}$, intensities up to $1 \mathrm{~mA}$ and within a pulse width of less then $10 \mathrm{ps}$. With these parameters the electron beam is used also to provide high intensity neutron beams, by stopping the electrons in a liquid lead target, which is $1.22 \mathrm{~cm}$ long ( 2 radiation lengths) and enclosed by a thin $(0.05 \mathrm{~cm})$ molybdenum channel: on such radiator bremsstrahlung photons produce neutrons through $(\gamma, \mathrm{n})$ reactions. Behind the radiator, a dump in aluminum surrounded with a lead shield acts as absorber of the electron beam and of a large fraction of the bremsstrahlung produced on the lead target. A view of the photo-neutron source close to the target is shown in fig. 1. Since the photoproduced neutrons have an isotropic spatial distribution, a high suppression of the electrons and photons is obtained by selecting neutrons far from the forward peak of the bremsstrahlung: the neutron beam is then actually defined at $95.5^{\circ}$ respect to the electron beamline, through a collimator with a borated polyethylene/lead structure inserted in a concrete wall, $2.4 \mathrm{~m}$ deep. After the collimator the neutron beamline enter the experimental room, where after several meters available for time-of-flight measurements is stopped by a final dump, which

This is an Open Access article distributed under the terms of the Creative Commons Attribution-Noncommercial License 3.0, which permits unrestricted use, distribution, and reproduction in any noncommercial medium, provided the original work is properly cited. 

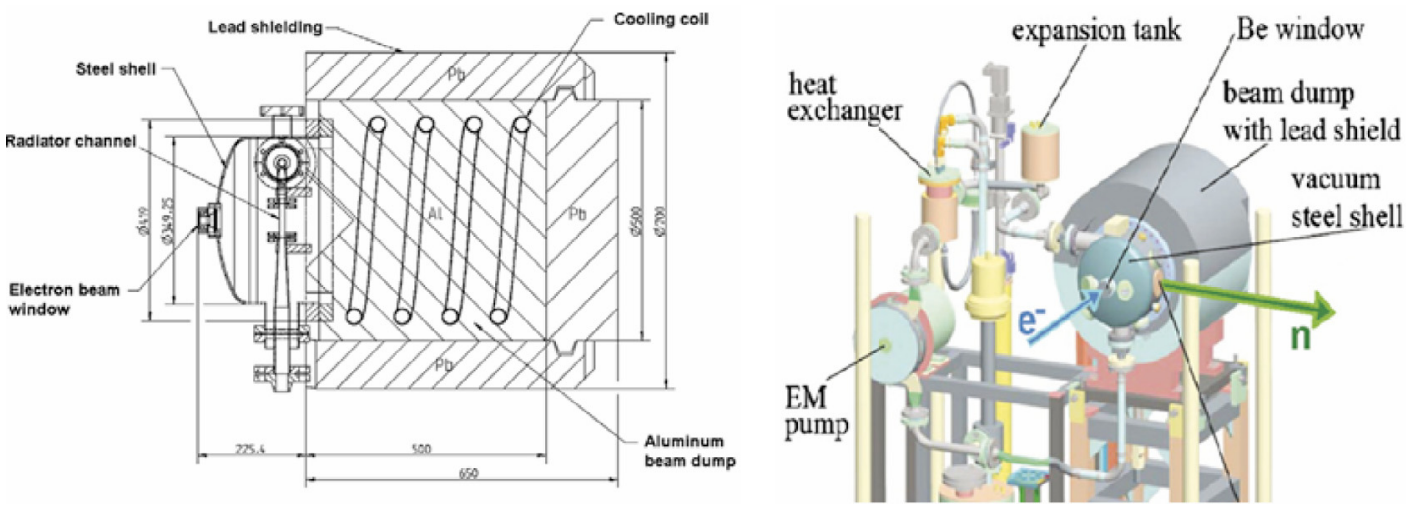

Fig. 1. A detailed view of the target area: the radiator channel containing the liquid lead, the steel shell confining a vacuum volume close to the target and the aluminum-lead dump are visible.

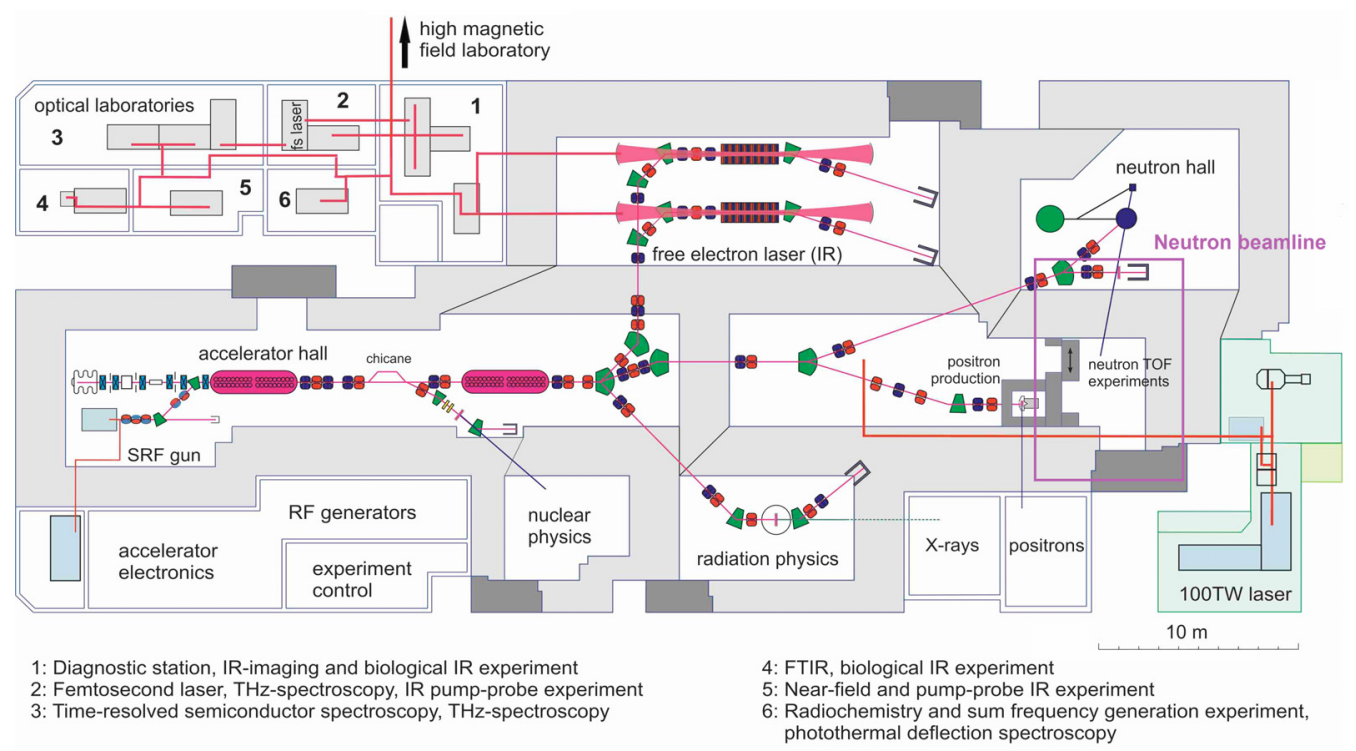

Fig. 2. Sketch of the ELBE accelerator complex. In the right side is visible the present neutron hall with the photo-neutron source and the collimator channel toward the neutron TOF experimental room.

is positioned close to the final wall and is made in borated polyethylene, enveloped by a thin layer of cadmium, and lead. A sketch of the present neutron beamline in the ELBE accelerator complex is visible in fig. 2, while detailed references can be found in $[2,3]$.

The primary physics goal of the nELBE neutron beamline is to measure fast neutron cross sections relevant for the nuclear transmutation of long-lived radioactive nuclides and of interest for GEN IV reactors. The measurement program is based on the NEA high priority list of target accuracies for fast reactors (see tab. 32 in [4]). The list includes requests for neutron cross sections between about $25 \mathrm{keV}$ and $20 \mathrm{MeV}$, which is the relevant energy range at nELBE.

\section{The new nELBE photo-neutron beamline}

The new neutron experimental room dedicated to the measurements of fast neutron cross sections for transmutation studies will be built at FZD in the next years, as a part of the expansion project of 


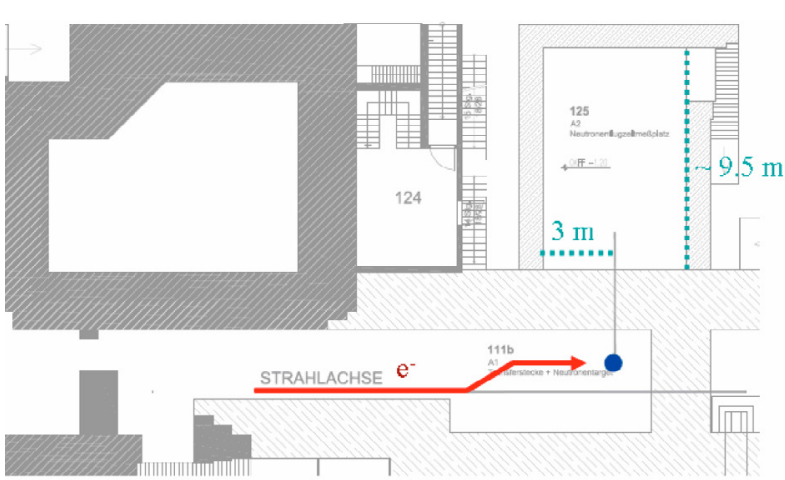

(a)

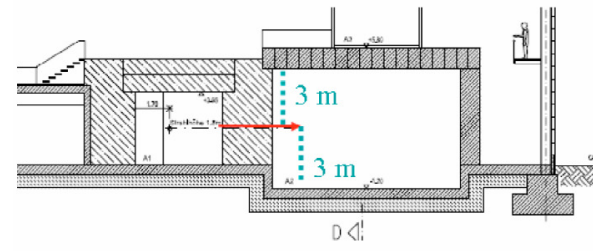

(b)

Fig. 3. Sketch of the new neutron beamline: (a) the new designed electron line toward the liquid lead radiator is visible, together with the new experimental room; (b) lateral view.

the ELBE facility. The goal is the creation of a national center for high power radiation sources, the $\mathrm{X}$-ray source using laser-Compton backscattering and the high power petawatt laser for ion acceleration being the other two key research areas.

A sketch of the new neutron beamline is showed in fig. 3. The electron beam will have an enhanced energy up to $50 \mathrm{MeV}$ and will hit the photo-neutron source travelling along a line at $1.8 \mathrm{~m}$ from the floor $(40 \mathrm{~cm}$ higher respect to the normal electron line). After $1 \mathrm{~m}$ flight the selected neutron beam will enter the collimator inserted in a heavy concrete wall $\left(52 \% \mathrm{Fe}\right.$, density $\left.3.6 \mathrm{~g} / \mathrm{cm}^{3}\right)$, which will be $2.5 \mathrm{~m}$ long. The beam will enter then the experimental room, which will be considerably larger then the actual one and therefore less affected from the background due to the neutron scattering in the room. Moreover, a longer flight path (the room is about $9.5 \mathrm{~m}$ long) will be available for TOF measurements. The walls of the experimental room, as well as the roof, will be in normal concrete (density $2.6 \mathrm{~g} / \mathrm{cm}^{3}$ ) while all the walls around the photo-neutron source will be in heavy concrete and $2.5 \mathrm{~m}$ long, as the one containing the collimator.

In order to perform an optimization study of the new line, as well as to satisfy all the radiation protection requirements (an accurate shielding study can be found in [5]), the complete simulation of the photo-neutron beamline has been performed with the FLUKA Monte Carlo code [6, 7], version 2008.3c. Starting from the photoproduction process, we included the accurate description of the photoneutron source, the shielding walls (lateral walls, floor and roof), the collimator, the experimental room and the final dump at the end of the beamline. For the electron beam the most critical operation conditions ( $1 \mathrm{~mA}$ current, $50 \mathrm{MeV}$ energy) have been assumed.

\section{Characterization of the radiation fields}

As first step, the radiation fields due to the neutron and photon components coming from the liquid lead radiator have been fully characterized. In fig. 4 are shown the neutron and the photon spectra that are calculated by integrating over all the solid angle the particle current exiting from the radiator. They are obtained by considering only the photoproduction target, at this level without the dump simulation: their integral gives, therefore, the total neutron and photon yield of the source. We have obtained: $n_{\text {yield }}($ tot $)=5.6710^{-3}$ neutrons $/ \mathrm{e}^{-}, \gamma_{\text {yield }}($ tot $)=6.68 \gamma / \mathrm{e}^{-}$. At the entrance of the collimator (i.e. at $1 \mathrm{~m}$ from the source) we estimate: $n_{\text {yield }}=4.3410^{-8}$ neutrons $\mathrm{cm}^{-2}$ per primary $\mathrm{e}^{-}$.

\subsection{Strength of the enhanced source and FLUKA/MCNP comparison}

From the calculated total neutron yield the total strength of the enhanced neutron source at $1 \mathrm{~mA}$ current can be estimated: $3.54 \cdot 10^{13}$ neutrons/s. With the aim to cross-check the results, a comparison 

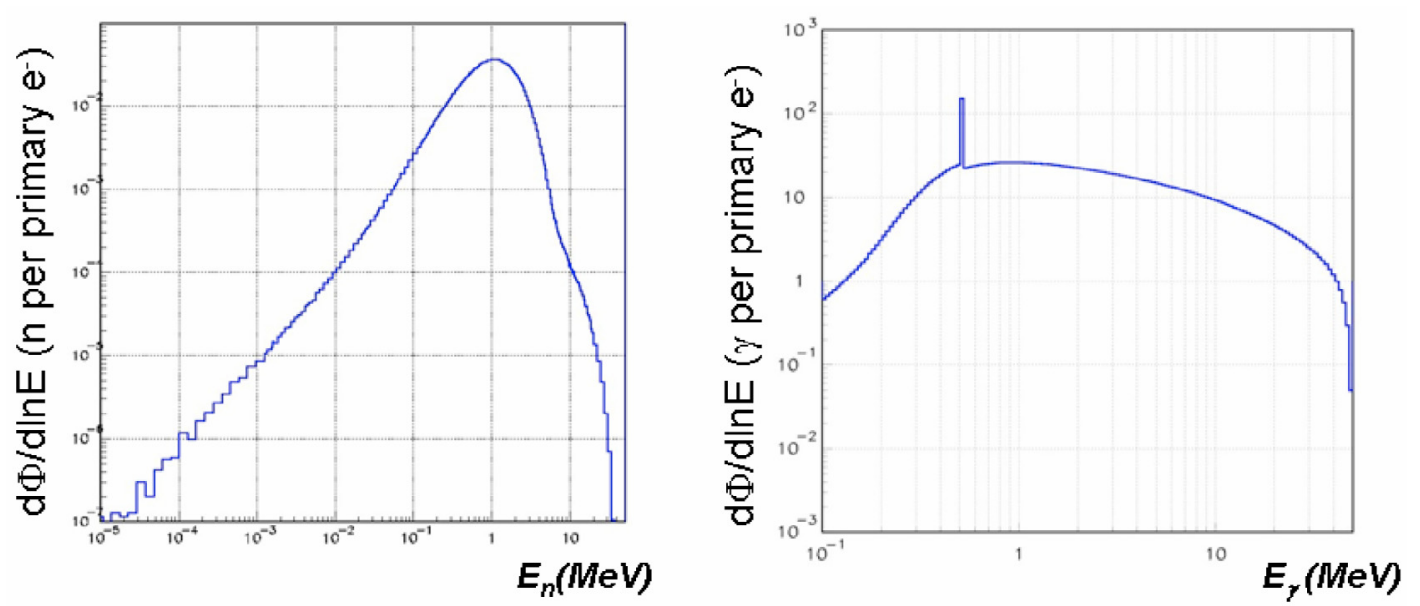

Fig. 4. Neutron and photon spectra from the liquid lead target, integrated over the whole solid angle. An ideal source is considered, without the beam dump beyond the radiator.

Table 1. FLUKA/MCNP source strength comparison at different energies of primary electrons.

\begin{tabular}{|c|c|c|c|}
\hline $\begin{array}{c}\text { Electron energy } \\
(\mathrm{MeV})\end{array}$ & $\begin{array}{c}\text { Neutron yield } \\
\left(\text { neutrons/ } \mathrm{e}^{-} \text {) }\right.\end{array}$ & \multicolumn{2}{|c|}{$\begin{array}{c}\text { Neutron source strength at the radiator } \\
\text { (neutrons/s) }\end{array}$} \\
\hline & FLUKA & MCNP [2] & FLUKA \\
\hline 20 & $1.20510^{-3}$ & $7.910^{12}$ & $7.5210^{12}$ \\
\hline 30 & $3.10810^{-3}$ & $1.910^{13}$ & $1.9410^{13}$ \\
\hline 40 & $4.5110^{-3}$ & $2.710^{13}$ & $2.8110^{13}$ \\
\hline 50 & $5.6710^{-3}$ & & $3.5410^{13}$ \\
\hline
\end{tabular}

with the nELBE published results [2] obtained with the MCNP code has been done at energies ranging between 20 and $40 \mathrm{MeV}$. The table 1 shows the comparison, which exhibits a very satisfactory agreement at the level of few percent. The data obtained with the FLUKA code have a statistical accuracy $\leq 1 \%$.

\subsection{Cross-check with the Swanson semi-empirical formula}

An additional cross-check has been done with the Swanson equation [8]:

$$
n_{\text {yield }}=9.3 \cdot 10^{10} Z^{(0.73 \pm 0.05)} \text { neutrons } s^{-1} k W^{-1}
$$

where the neutron yield is expressed as a function of the atomic number of the target material and the incident beam power. The semi-empirical formula (1) has been derived by folding the experimental photo-neutron cross sections with a numerical integration of the photon track length distributions coming from the analytical theory of the showers, and is valid at high energies and for semi-infinite targets. For the asymptote of the lead curve we find: $2.32 \cdot 10^{12}$ neutrons $\mathrm{s}^{-1} \mathrm{~kW}^{-1}$. By using the Swanson curves that can be found in [8] to correct the energy effect, and a dedicated simulation to find the correction factor for the finite dimensions of the slab ( 2 radiation lengths in our case), we estimate: $1.92 \cdot 10^{13}$ neutrons s${ }^{-1}$ at $30 \mathrm{MeV}$ and with $1 \mathrm{~mA}$ current, in very good agreement both with MCNP and FLUKA. 
Table 2. Neutron/photon ratio at the exit of the collimator for different choices of the collimator orientation respect to the electron beamline, in the case of an ideal source (only target) and a real one (target and dump).

\begin{tabular}{|l|c|c|c|c|c|c|}
\hline & $\mathbf{9 0}^{\circ}$ & $\mathbf{9 5 . 5}^{\circ}$ & $\mathbf{1 0 0}^{\circ}$ & $\mathbf{1 0 5}^{\circ}$ & $\mathbf{1 1 0}^{\circ}$ & $\mathbf{1 1 5}^{\circ}$ \\
\hline $\begin{array}{l}\text { Ideal source } \\
\mathrm{n}_{\text {yield }} / \gamma_{\text {yield }}\end{array}$ & $2.20 \cdot 10^{-3}$ & $2.38 \cdot 10^{-3}$ & $2.52 \cdot 10^{-3}$ & $2.77 \cdot 10^{-3}$ & $2.88 \cdot 10^{-3}$ & $3.05 \cdot 10^{-3}$ \\
\hline $\begin{array}{l}\text { Real source } \\
\mathrm{n}_{\text {yield }} / \gamma_{\text {yield }}\end{array}$ & $2.17 \cdot 10^{-3}$ & $2.39 \cdot 10^{-3}$ & $2.53 \cdot 10^{-3}$ & $2.78 \cdot 10^{-3}$ & $2.92 \cdot 10^{-3}$ & $3.14 \cdot 10^{-3}$ \\
\hline
\end{tabular}
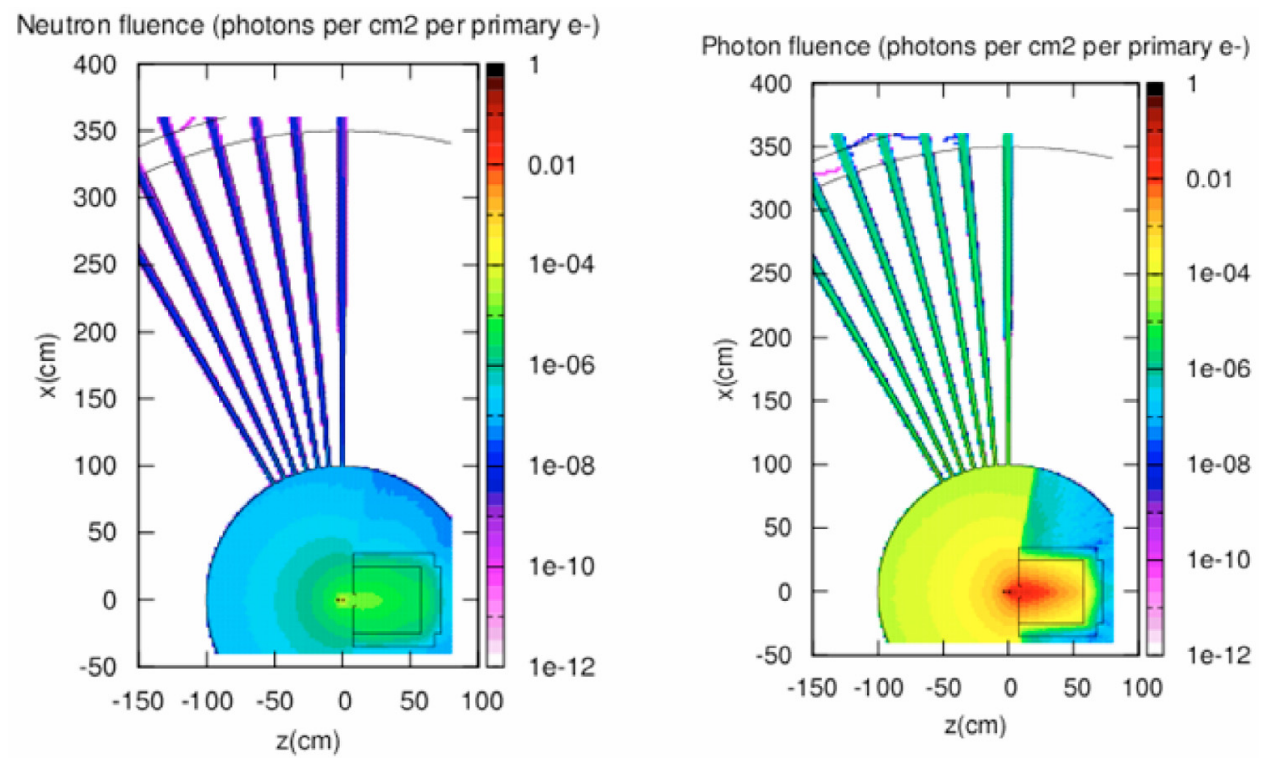

Fig. 5. Neutron and photon fluences (part $\mathrm{cm}^{-2}$ per primary $\mathrm{e}^{-}$) around the photo-neutron source with several choices of the collimator line, in the case of a real source.

\section{The optimization of the neutron beamline direction}

A key point of the design of the new photo-neutron source is the optimization of the neutron beamline direction: taking into account the isotropy of the neutron production and the typical shape of the bremsstrahlung, the ratio $\mathrm{n}_{\text {yield }} / \gamma_{\text {yield }}$ can be maximized. The optimized direction will be implemented in the new neutron beamline by rotating the whole photo-neutron source (the liquid lead target and the dump). With this goal a dedicated simulation has been done (see fig. 5), by looking at the neutron/photon ratio at the exit of several collimators, oriented at different angles in the range $\left[90^{\circ}, 115^{\circ}\right]$. Only the collimator channels have been described, considering the materials around as infinite absorbers: all the analyzed directions have been then studied in the same simulation. Moreover, since in the real experimental arrangement we have to avoid a possible contamination coming from the neutrons that are scattered on the beam dump or photoproduced in the dump material, two simulations have been performed: one with the ideal case of the radiator without dump and a second one with the liquid lead target and the dump. The results are reported in table 2 : by moving the angle from $90^{\circ}$ to $115^{\circ}$ the neutron/photon ratio increases of about $40 \%$. The contamination starts to be visible only at $110^{\circ}(\sim 1.4 \%)$ and at $115^{\circ}$ is still at the acceptable level of $3 \%$.

\section{Conclusions}

In this paper has been presented the study done to assess some optimization aspects of the new nELBE neutron beamline that will be realized in the next years at Forschungszentrum Dresden-Rossendorf. 


\section{EPJ Web of Conferences}

The higher intensity of the source, the large dimensions of the experimental room, the very satisfactory shielding conditions and an optimized orientation of the neutron beamline will allow us to perform measurements with a better statistics, less affected by background and with an enhanced neutron/photon ratio.

\section{References}

1. J. Teichert, A. Büchner, P. Evtushenko, F. Gabriel, U. Lehnert, P. Michel, J. Voigtländer, Results of beam parameter measurement of the ELBE electron accelerator after commissioning, NIM A 507, 354-356 (2003).

2. E. Altstadt, C. Beckert, H. Freiesleben, V. Galindo, E. Grosse, A.R. Junghans, J. Klug, B. Naumann, S. Schneider, R. Schlenk, A. Wagner, F.-P. Weiss, A photo-neutron source for timeof-flight measurements at the radiation source ELBE, Ann. of Nucl. En. 34, 36-50 (2007).

3. J. Klug, E. Altstadt, C. Beckert, R. Beyer, H. Freiesleben, V. Galindo, E. Grosse, A.R. Junghans, D. Légrády, B. Naumann, K. Noack, G. Rusev, K.D. Schilling, R. Schlenk, S. Schneider, A. Wagner, F.P. Weiss, Development of a neutron time-of-flight source at the ELBE accelerator, NIM A 577, 641-653 (2007).

4. International Evaluation Co-operation of the NEA Nuclear Science Committee, Uncertainty and target accuracy assessment for innovative system using recent covariance data evaluations, NEAWPEC-26 (2008), http://www.nea.fr/html/science/wpec/volume26/volume26.pdf

5. Anna Ferrari, R. Beyer, E. Birgersson, J. Claussner, E. Grosse, R. Hannaske, A. Junghans, T. Kögler, I. Kösterke, R. Massarczyk, A. Matič, K.-D. Schilling, G. Schramm, R. Schwengner, A. Wagner and F.P. Weiss, Shielding aspects of the new nELBE photo-neutron source, Proceedings of the SATIF-10 Workshop, CERN 2-4 June 2010.

6. G. Battistoni, S. Muraro, P.R. Sala, F. Cerutti, A. Ferrari, S. Roesler, A. Fasso', J. Ranft, The FLUKA code: description and benchmarking, Proceedings of the Hadronic Shower Simulation Workshop 2006, Fermilab 6-8 September 2006, M. Albrow, R. Raja editors, AIP Conference Proceeding 896, 31-49 (2007).

7. A. Fasso, A. Ferrari, J. Ranft and P.R. Sala, FLUKA: a multi-particle transport code, CERN-200510 (2005), INFN/TC_05/11, SLAC-R-773.

8. W.P. Swanson, Calculation of neutron yields released by electrons incident on selected materials, SLAC-PUB-2042 (1978). 